\title{
FRACTURE OF THE NECK OF THE RADIUS WITH MEDIAL DISPLACEMENT OF THE HEAD
}

\author{
Report of Three Cases
}

\author{
M. H. El Ghawabi, Cairo, Egypt \\ From the Ain Shams University, Cairo
}

This paper describes three cases in all of which the radial head or fragments of it were excised through a medial incision.

\section{CASE REPORTS}

Case 1-A woman aged fifty-two was knocked down by a car and fell on her outstretched left hand. There was severe pain in the left elbow and she could move it only with difficulty. On examination the elbow was markedly swollen especially on the medial side. There was terminal limitation, with pain, of extension, supination and pronation. There was local tenderness on the lateral side of the joint, where the radial head could not be felt in its normal site, but it was felt at the medial side of the elbow. Radiographs showed a fracture of the neck of the radius with displacement of the radial head to the antero-medial aspect of the proximal ulna (Fig. 1). Its upper articular surface was directed downwards and medially. The displaced head showed a fracture in its middle with minimal displacement of its two nearly equal fragments. A small fragment from the neck was seen in the radiohumeral joint above the radial shaft. There was no subluxation of the elbow joint.

Treatment and progress-The radial head was excised through a medial incision. It was lying in the flexor digitorum profundus: a track filled with organising blood clot led to the humero-radial joint. The track passed deep to the contents of the cubital fossa through the deep fibres of the brachialis muscle.

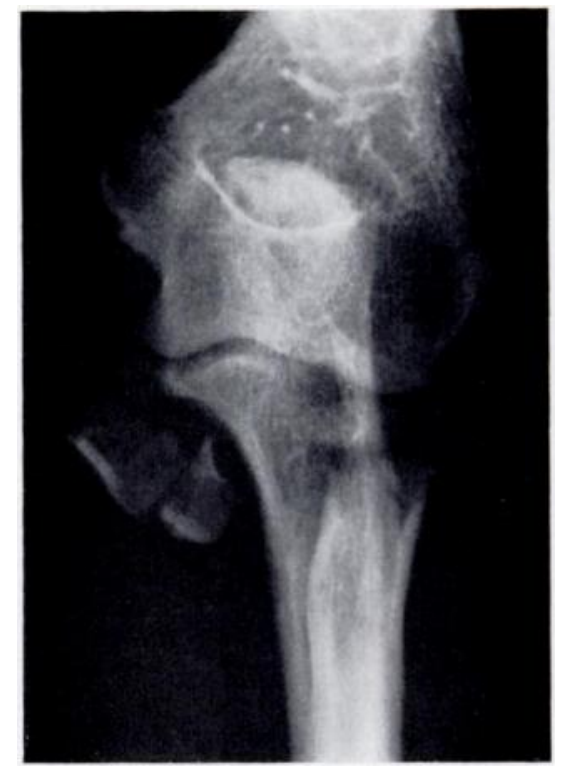

FIG. 1

Case 1-Radiograph showing the displacement of the radial head.

The elbow was protected in plaster for three weeks, and thereafter active exercises were begun. Review ten years later showed marked limitation of supination and pronation. There was slight extension. Radiographs showed ossification around the radial stump and along the medial border of the upper part of the ulna.

Case 2-A man aged twenty-two injured his left elbow in a fall from a ladder. The clinical picture was similar to that in the previous case apart from an associated partial ulnar nerve lesion. There was no vascular complication. Radiographs showed an oblique fracture of the radial neck with complete displacement of the head to a new position medial and anterior to the upper part of the ulna (Figs. 2 and 3). Its articular surface was directed posteriorly and slightly upwards. The head was fractured but without displacement of its fragments.

At operation on the next day the radial head was excised through a medial incision. It was found in the flexor digitorum profundus. A track full of blood clot led to the elbow 
joint. Through this track the remaining fragments were removed from the elbow joint by a small curette. The limb was supported in plaster for three weeks. Review after a year showed marked limitation of supination and pronation as well as of the terminal degrees of extension.

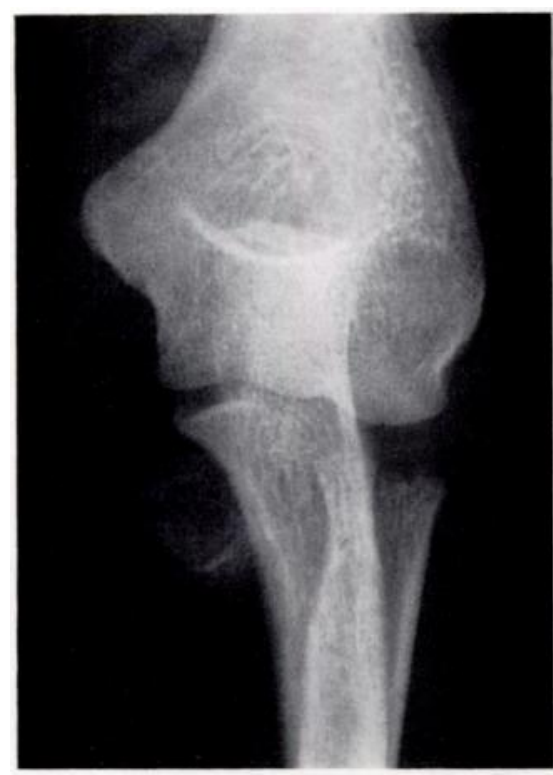

Fig. 2

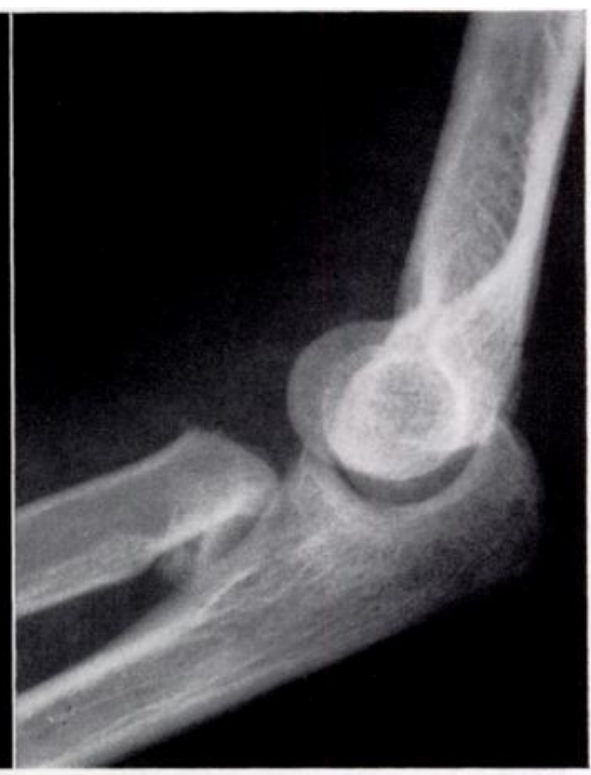

FIG. 3

Case 2-Antero-posterior and lateral radiographs of the elbow.

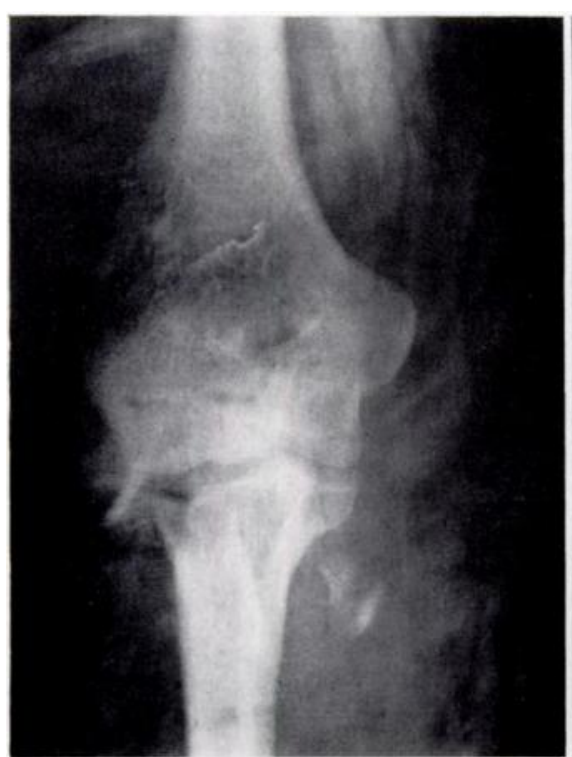

FIG. 4

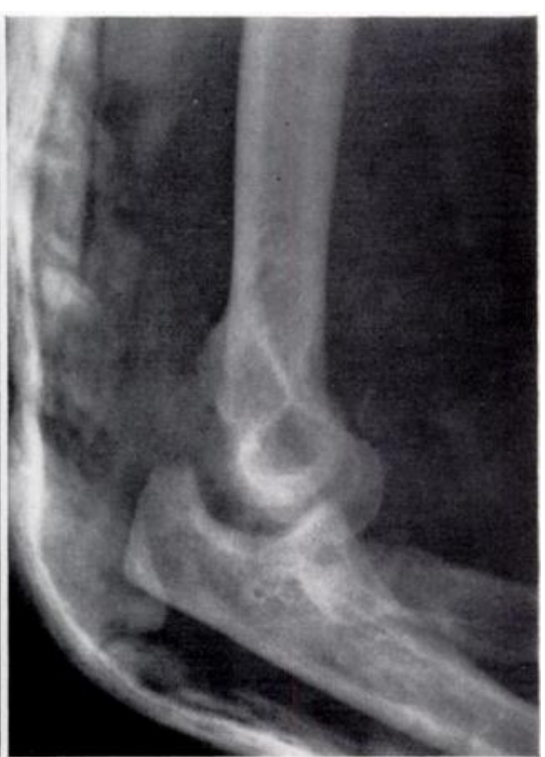

FIG. 5

Case 3-Antero-posterior and lateral radiographs to show the displacement of half the radial head.

The ulnar nerve had recovered completely. Radiographs showed ossification around the radial stump, along the medial side of the elbow, and at the site from which the radial head had been excised, medial to the ulna. 
At a further operation the stump of the radius was trimmed and excess bone was removed. This gave improvement in the range of supination and pronation but the limitation of extension persisted.

Case 3-A man aged thirty-five injured his right elbow in a fall on the outstretched hand with a valgus strain of the elbow. He described forcible supination of the right forearm in the fall, and had felt a sharp painful snap in the elbow. Clinical examination revealed posterior dislocation of the right elbow with a painful swelling over the radial head and over the medial border of the cubital fossa. There was no nerve injury. Radiographs confirmed the dislocation and showed a comminuted fracture of the radial head. One half of the radial head was displaced to the front and medial surface of the forearm while the other half remained in its place (Figs. 4 and 5). Immediate closed reduction of the dislocation was done under general anaesthesia and fixation in an above-elbow plaster was kept on for three weeks; then the radial fragments were excised. At operation the fragment lying medially was removed from the substance of the flexor digitorum profundus where it was close to the ulnar nerve. There was a track lined by granulation tissue leading to the elbow joint across the deep fibres of the brachialis muscle. Through a separate lateral incision the other half of the radial head was excised. A plaster cast was applied and kept on for another three weeks before active exercises were begun.

Examination fourteen months later revealed limitation of the terminal degrees of extension; flexion, supination and pronation were nearly full.

\section{DISCUSSION}

Fracture of the neck of the radius with medial displacement of the head is rare. WatsonJones (1956) mentioned three cases, in one of which the pressure of the displaced fragment caused ulnar nerve paralysis.

A study of our three cases and of other reported cases suggests that there may be two types of this injury. In the first type the whole head is displaced medially without dislocation of the elbow, as in Cases 1 and 2. In the second type there is associated posterior elbow dislocation; the radial head is fragmented and some of the fragments are displaced medially while the rest of the fragments remain laterally as in our Case 3 and in one case described by Watson-Jones (1955). In this second type of case trauma is unusually severe. Apart from the indirect compression forces an associated severe valgus strain could cause the medial structures of the elbow to tear thus predisposing to the ossification seen later. In Case 3 forcible supination was an added factor. Medial displacement of the head was facilitated by the associated dislocation of the elbow where some of the fragments of the head were thrust medially among the flexors. As regards the first type, it is uncertain whether a momentary dislocation of the elbow occurs. A possible explanation for the medial displacement is that the radial head is squeezed out between the capitulum and the stump of the radius.

\section{SUMMARY}

1. Three cases of fracture of the neck of the radius with medial displacement of the head are described.

2. It is proposed that such injuries be classified into two types: 1) displacement of the radial head without dislocation of the elbow; and 2) displacement during posterior dislocation of the elbow.

\section{REFERENCE}

Watson-Jones, R. (1955): Fractures and Joint Injuries. Fourth edition. Volume 2, p. 554. Edinburgh and London: E. \& S. Livingstone Ltd. 